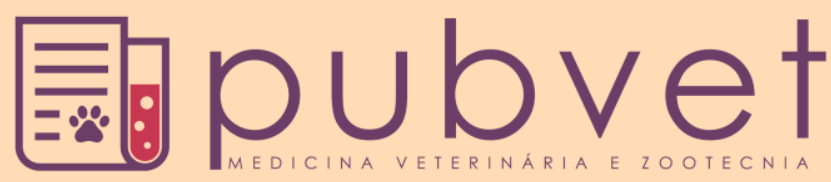

https://doi.org/10.22256/pubvet.v12n7a123.1-8

\title{
Hydra (Cnidaria, Hydrozoa) como modelo em estudos de ecotoxicidade: Revisão
}

\author{
Marlos de Medeiros Chaves ${ }^{1 * \bullet}$, Maria Cláudia Colla Ruvolo-Takasusuki ${ }^{2} \bullet$ \\ ${ }^{1}$ Tecnologista da Fundação Oswaldo Cruz, Fortaleza, Ceará, Brasil. E-mail: marlosfiocruz@gmail.com \\ ${ }^{2}$ Professora Associada da Universidade Estadual de Maringá, Departamento de Biotecnologia, Genética e Biologia Celular, Maringá, Paraná, \\ Brasil. E-mail : mccrtakasusuki@uem.br \\ *Autor para correspondência
}

\begin{abstract}
RESUMO. O processo de industrialização e o crescimento acelerado da população mundial que ocorreu nas últimas décadas colocaram em risco diversos ecossistemas, em especial os aquáticos, que tiveram contato com numerosas substâncias químicas potencialmente tóxicas. Estudos de ecotoxicidade com organismos sensíveis a pequenos desequilíbrios ambientais são estudados de forma crescente com o objetivo de testar substâncias que poderiam representar efeitos tóxicos no organismo humano. Dentro deste grupo, destacam-se os pequenos cnidários do gênero Hydra. Por possuir uma série de características favoráveis, este grupo se consolidou como organismo-teste frente a diversos tipos de espécies. Este trabalho de revisão teve o propósito de reunir as informações mais relevantes sobre a importância dos testes de ecotoxicidade e as vantagens e desvantagens da utilização de espécies do gênero Hydra em tais estudos.
\end{abstract}

Palavras chave: Bioensaios, ecotoxicologia, hydra, organismo-teste

\section{Hydra (Cnidaria, Hydrozoa) as a model in ecotoxicity studies: Review}

\begin{abstract}
The industrialization process and the accelerated growth of the world population that it happened in the last decades put in risk several ecosystems, especially the aquatic ones that had contact with numerous potentially toxic chemical substances. Ecotoxicity studies with organisms sensitive to small environmental imbalances are increasingly being studied with the aim of testing substances that could represent toxic effects on the human body. Within this group, the small cnidarians of the genus Hydra stand out. By having a series of favorable characteristics, this group consolidated as test organism in front of several species types. This review work had the purpose of gathering the most relevant information about the importance of the tests of ecotoxicity and the advantages and disadvantages of the use of species of the genus Hydra in such studies.
\end{abstract}

Keywords: Bioassays, ecotoxicology, hydra, test organism

\section{Hydra (Cnidaria, Hydrozoa) como modelo en estudios de ecotoxicidad: Revisión}

RESUMEN. El proceso de industrialización y el crecimiento acelerado de la población mundial que ha ocurrido en las últimas décadas han puesto en riesgo diversos ecosistemas, en particular los acuáticos, que han tenido contacto con numerosas sustancias químicas potencialmente tóxicas. Los estudios de ecotoxicidad con organismos sensibles a pequeños desequilibrios ambientales se estudian de forma creciente con el objetivo de probar sustancias que podrían representar efectos tóxicos en el organismo humano. Dentro de este grupo, se destacan los pequeños cnidarios del género Hydra. Por poseer una serie de 
características favorables, este grupo se consolidó como organismo-prueba frente a diversos tipos de especies. Este trabajo de revisión tuvo el propósito de reunir las informaciones más relevantes sobre la importancia de las pruebas de ecotoxicidad y las ventajas y desventajas del uso de especies del género Hydra en tales estudios.

Palabras clave: Bioensayos, ecotoxicología, hydra, organismo-prueba

\section{Introdução}

A modernização e aperfeiçoamento dos processos de industrialização da sociedade levou ao desenvolvimento de novos materiais que trouxeram avanços significativos nas diversas áreas da Ciência, objetivando melhorar a qualidade de vida do ser humano. Entretanto, o que realmente observamos, é que centenas destas substâncias acabam alcançando corpos hídricos sendo transportadas pelas chuvas (Ribeiro et al. 2007), ou sendo depositadas em locais inapropriados.

Estes poluentes, além de alterar a composição natural do meio, têm potencial para interferir no ecossistema, modificando a relação entre produtores e consumidores. Neste processo, as substâncias tóxicas são gradativamente absorvidas pelos organismos em um mecanismo de bioacumulação (Santos \& Silva 2007). De acordo com Rand et al. (1995), um tóxico é um agente capaz de produzir resultado prejudicial no sistema biológico, alterando sua estrutura ou função, ou pode também provocar a morte.

No organismo humano, já foram relatados diversos casos na medicina de efeitos carcinogênicos e disfunções no sistema nervoso em virtude do contato com substâncias industrializadas diversas (Trouiller et al. 2009; Ahamed et al. 2013; Raghunathan et al. 2013).

Com o intuito de estudar e avaliar de forma segura o potencial tóxico de substâncias, elementos químicos ou contaminantes em seres vivos ou ecossistemas, surgiu a Ecotoxicologia (Knie \& Lopes 2004).

A toxicidade a uma substância química está intrinsicamente relacionada com a sua concentração, suas propriedades químicas e o tempo de exposição (Rand et al. 1995). Para detectar ou avaliar os efeitos adversos ou não de uma ou mais substâncias sobre os sistemas biológicos, são utilizadas técnicas denominadas testes de toxicidade. Estes testes constituem-se basicamente na exposição de organismos a diferentes condições, as quais tentam simular o ambiente natural, visando assim a detectar seus efeitos letais e/ou subletais (Laitano 2003). Por meio destes testes, é viável o monitoramento do impacto provocado por agentes químicos em ecossistemas com precisão, além da natureza destes efeitos e o grau de toxicidade (Santos \& Silva 2007), constituindo assim uma valiosa ferramenta de controle. Os testes ecotoxicológicos podem ser utilizados para diversos setores, entre eles licenciamento de produtos químicos, fiscalização de efluentes, monitoramento de qualidade de águas, entre outros (Knie \& Lopes 2004). De acordo com Santos and Silva (2007) a seleção de um organismo para um estudo ecotoxicológico deve seguir alguns critérios: facilidade de reprodução; facilidade de cultivo; disponibilidade no mercado; facilidade de manipulação; velocidade de crescimento e desenvolvimento. Os organismos-teste são utilizados como indicadores em testes de toxicidade por possuírem uma reduzida tolerância a compostos químicos, podendo apresentar diversas alterações anatômicas, fisiológicas e comportamentais quando em contato com substâncias tóxicas (Magalhaes \& Ferrão Filho 2008). Diversos tipos de organismos podem ser utilizados como organismos-teste. Podemos destacar diversas espécies de algas, bactérias, invertebrados e peixes. A escolha leva em consideração fatores relacionados a: sensibilidade do organismo teste a um agente tóxico, capacidade de gerar descendentes geneticamente idênticos e em um ciclo de vida curto, fácil cultivo e pertinência ecológica (Santos \& Silva 2007). Uma das principais razões para a inclusão de invertebrados nos estudos de ecotoxicidade está no fato de que estes animais constituem a maioria em todos os ecossistemas e estão sempre em abundância (Fossi et al. 1998). A utilização de invertebrados aquáticos pode fornecer importantes evidências de danos químicos em ecossistemas (Herricks \& Cairns Junior 1982). No grupo dos invertebrados, linhas de pesquisa envolvendo diversos cnidários, e, em especial as hidras, vem sendo cada vez mais explorada, tendo em vista a crescente preocupação com a poluição e seus efeitos no meio ambiente (Trottier et al. 1997).

A hidra (gênero Hydra) é um animal invertebrado, pertencente ao filo Cnidária (classe Hydrozoa) medindo aproximadamente de 2 a 25 
$\mathrm{mm}$. Seu corpo tem o formato de pólipo, com uma das extremidades apoiado em algum substrato e na outra apresentando uma boca rodeada por longos tentáculos equipados com estruturas urticantes utilizados para capturar presas. Vivem em água doce e limpa em lagoas e açudes. Uma das propriedades mais fascinantes desses organismos, e que até hoje é razão de inúmeras publicações é a incrível capacidade regenerativa desses animais (Massaro \& Rocha 2008; Galliot 2012; Schaible et al. 2014). A utilização das hidras em testes ecotoxicológicos tem se tornado cada vez mais atraente devido a uma série de vantagens: (a) sofrem alterações gradual em sua morfologia em contato com toxinas; (b) habitam vários ecossistemas de água doce; (c) são facilmente cultivados e mantidos no laboratório; (d) tem a capacidade de se reproduzir rapidamente; (e) custo baixo na realização dos experimentos (Trottier $e t$ al. 1997).

O objetivo deste trabalho foi revisar na literatura científica a utilização de organismos do gênero Hydra como modelos de estudo em ecotoxicidade. Para isso, foram consultadas as bases de dados MEDLINE, LILACS e SciELO utilizando os termos "hydra" e "ecotoxicology". Foram utilizados artigos publicados de 1977 até 2017.

\section{Hidras: modelo para Ecotoxicologia}

Os ecossistemas aquáticos são submetidos constantemente a contaminantes que contribuem com estresse múltiplos em organismos que vivem nestes ambientes. $\mathrm{O}$ estudo do efeito destes poluentes sobre organismos aquáticos representa um esforço importante para a sua preservação. A medição apenas dos atributos físico-químicos da água não é o suficiente para avaliar a saúde de um ecossistema aquático (Ten Brink \& Woudstra 1991). O conceito de biomonitoramento parte da suposição de que a medição da saúde da biota pode ser usada para avaliar a saúde de um ecossistema (Herricks \& Cairns Junior 1982).

A hidra surgiu como um modelo experimental para a Biologia em 1741, quando o Naturalista Suíço Abraham Trembley descobriu não só a fantástica capacidade regenerativa desses exóticos organismos, mas também diversas outras aplicações experimentais. Nos últimos 270 anos, a hidra vem sendo amplamente utilizada para responder questões ligadas à Biologia Molecular, Neurofisiologia, Imunologia, Ecossistemas e Ecotoxicologia. No campo da Ecotoxicologia aquática, a hidra se consolidou como um modelo altamente satisfatório (Galliot 2012), ganhando crescente atenção como uma espécie-alvo sensível e bioindicador.

Tradicionalmente, a hidra vem sendo utilizada para avaliar a toxicidade aguda e regenerativa frente a metais, compostos orgânicos, produtos farmacêuticos e nano materiais. $\mathrm{O}$ uso de uma bateria de bio-ensaios para avaliação de amostras ambientais complexas tem sido amplamente recomendado frente a um único bio-ensaio, uma vez que é improvável que um único bio-ensaio responda a todos os possíveis tóxicos (Clarke et al. 1990). A sensibilidade da morfologia da hidra como um indicador da toxicidade sub letal e da rapidez com que os efeitos da taxa de crescimento da população podem ser observados, fazem da hidra espécies de teste de toxicologia de utilidade única (Quinn et al. 2012).

A aplicação dos testes de toxicidade pode variar dependendo do período de tempo de avaliação. Assim, temos os testes de toxicidade aguda, quando se pretende avaliar a letalidade e inércia de um determinado organismo em período curto de tempo (cerca de 24 a 48 horas) e os testes de toxicidade crônica, quando se pretende avaliar alterações fisiológicas diversas (reprodutiva, comportamental, crescimento etc.) em um longo período de tempo (Dias et al. 2006). O objetivo dos testes é determinar a concentração letal para $50 \%$ dos organismos-testes de uma amostra por um período de exposição (CL50) (Santos \& Silva 2007).

A hidra oferece várias vantagens potenciais na análise dos efeitos tóxicos dos nano materiais devido ao seu pequeno tamanho, arquitetura do corpo simples, modo de reprodução assexuada, transparência do tecido e disponibilidade de protocolos confiáveis que permitem análises toxicológicas em todo o animal, a nível celular e molecular (Marchesano et al. 2015).

\section{Sensibilidade a metais}

As hidras são organismos sensíveis a metais pesados (zinco, cobre, urânio, magnésio, cádmio), possivelmente por não possuírem a proteína de ligação de metal metalotioneína (Galliot 2012). Em um estudo de Zeeshan et al. (2017) foi investigado a toxicidade ao cobalto em um modelo de hidra. Embora o cobalto seja um poluente ambiental conhecido, existe relativamente pouca informação sobre a sua toxicidade em animais aquáticos, especialmente cnidários. Os poucos 
animais aquáticos que foram estudados neste aspecto incluem peixes e invertebrados, como os crustáceos cladóceros (Classe Branchipoda), copépodes (Classe Maxillopoda), gamarídeos (Classe Malacostraca) e rotíferos (Kim et al. 2006). Esses estudos incidiram sobre toxicidades agudas e crônicas, incluindo efeitos sobre fatores relacionados à reprodução, regeneração e comportamento. $\mathrm{O}$ exame microscópico da hidra revelou um evidente padrão de mudanças estruturais. A primeira mudança observada foi a alteração no formato dos tentáculos seguido do seu encurtamento, bem como do seu corpo. A injúria progressiva levou a mudanças letais, como o arredondamento do corpo sem tentáculos visíveis, perda osmótica e desintegração total do corpo. A desintegração começou no disco do pedal e avançou rapidamente para o resto do corpo. Ensaios adicionais demonstraram ainda que o cobalto desencadeou apoptose mediada por mitocôndrias (Zeeshan et al. 2017). Em testes de toxicidade aguda avaliando a toxicidade do cobre, cádmium e zinco, Karntanut and Pascoe (2002) constaram efeitos tóxicos destes metais utilizando Hydra vulgaris, $H$. oligactis e H. viridissima. Em concentrações intermediárias destes metais, as hidras sofreram modificações no tamanho e formato dos tentáculos.

Os efeitos agudos dos metais nas hidras são baseados na letalidade estabelecidos pelas modificações morfológicas. Outra característica que é sensivelmente afetada é a reprodução assexuada destes animais. Os efeitos no crescimento de populações de hidra foram observados em uma concentração entre 8 e 16 $\mathrm{mg} / \mathrm{L}$ para o cobre (Pollino \& Holdway 1999; Karntanut \& Pascoe 2005), 0,8 mg/L para o cádmio (Holdway et al. 2001), $75 \mathrm{mg} / \mathrm{L}$ para o zinco (Holdway et al. 2001) e $50 \mathrm{mg} / \mathrm{L}$ para chumbo (Browne \& Davis 1977) em Hydra viridissima e $60 \mathrm{mg} / \mathrm{L}$ para níquel em Hydra littoralis (Santiago-Fandino 1983). Contudo, como destacado por Holdway et al. (2001), a toxicidade do metal é grandemente modificada pelos fatores abióticos, como a dureza da água, $\mathrm{pH}$ e temperatura utilizada pelos pesquisadores em vez de apenas diferenças de espécies. A alta dureza da água tende a aumentar a complexação de metais, bem como fornece cátions concorrentes $(\mathrm{Ca} 2+\mathrm{e} \mathrm{Mg} 2+)$ que podem diminuir os efeitos dos metais divalentes tóxicos (Riethmuller et al. 2001). Um detalhe que chama a atenção em estudos de sensibilidade com metais foi realizado por Pollino and Holdway (1999). O aumento da sensibilidade aguda ao cobre da espécie Hydra viridissima contendo algas simbiontes foi significativamente maior. Isto porque o cobre é considerado um potente algicida, afetando assim as Zoochlorella. No entanto, Karntanut and Pascoe (2005) compararam a toxicidade de metais pesados tanto em Hydra viridissima com e sem algas endossimbíticas. Embora a toxicidade fosse semelhante para ambos os grupos, nas concentrações inferiores de cobre as hidras simbióticas foram mais capazes de tolerar a toxicidade. Eles hipotetizaram que a baixas concentrações, o cobre absorvido pelas hidras simbióticas pode ser sequestrado pelas algas, proporcionando um grau de proteção para a própria hidra. No entanto, nas concentrações mais elevadas é provável que qualquer sistema de defesa esteja sobrecarregado pela toxina. Assim, o efeito de qualquer vantagem derivada da alga endosimbiótica é de pouca importância.

\section{Sensibilidade a nano partículas}

Os efeitos tóxicos do carvão ativado modificado com nano partículas de prata (AgNP) também foram testados usando espécimes de Hydra attenuata em trabalho de Gonçalves et al. (2016). A exposição causou alterações morfológicas observadas inicialmente nos tentáculos, resultando em seu encurtamento e em seguida a contração do corpo. Estas mudanças aconteceram rapidamente quando utilizado altas concentrações e tardiamente quando em baixas concentrações. A osmo-regulação da hidra foi prejudicada causando inchaço do pólipo e eventual morte e desintegração do animal em doses mais elevadas.

A exposição da hidra a nano partículas de óxido de cobre $(\mathrm{NPCuO})$ resultou em alterações morfológicas severas em trabalho publicado por Murugadas et al. (2016). As nano partículas NPCuO são geralmente empregadas na composição de tintas anti incrustante usadas em embarcações. Neste estudo, o autor submeteu espécies de hidra a experimentos com diferentes concentrações e intervalo de tempo. A exposição da hidra resultou em alterações morfológicas severas, dependendo da duração do ensaio. A perda da capacidade de alimentação, crescimento e regeneração foi também observada. As análises in vivo e in vitro revelaram indução de estresse oxidativo, genotoxicidade e morte celular apoptótica, acompanhada de interrupção da progressão do ciclo celular. 
Os efeitos toxicológicos do carbono puro multicamadas e quimicamente modificado denominado "carbon nano-onions" (CNOs) sobre o desenvolvimento do pólipo de água doce Hydra vulgaris foram investigados para elucidar os efeitos ecotoxicológicos dos CNOs (Marchesano et al. 2015). Os CNOs possuem extraordinárias propriedades físicas de crescente interesse para a indústria biomédica e eletrônica. Apesar dos inúmeros testes visando avaliar a toxicidade dos nano materiais, a ausência geral de efeitos adversos induzidos por CNOs sobre toxicidade a curto e longo prazo na hidra sugerem um grau razoável de biossegurança desta nova classe de materiais. Apesar da escassez de dados sobre a toxicidade do CNO, estes dados são coerentes com parte da literatura atual que consagra nanoestruturas à base de carbono (por exemplo, fullerenos) como materiais seguros em vários modelos biológicos.

\section{Sensibilidade a compostos farmacêuticos}

$\mathrm{O}$ lançamento de contaminantes farmacêuticos em efluentes também ocorre em uma frequência grande, causando uma grande preocupação ambiental. Esses produtos estão presentes normalmente em baixas concentrações, podendo causar efeitos crônicos mais relevantes (Fent et al. 2006). Entretanto, efeitos sub letais de fármacos misturados podem ocorrer, indicando que eles podem agir de forma aditiva em Hydra attenuata (Quinn et al. 2009). Em estudo desenvolvido por Pascoe et al. (2003) foram testados 10 tipos de medicamentos. Três deles (diazepan, amlodipina e digoxina) inibiram a regeneração de hidras após um período prolongado de exposição (17 dias).

Estudos com o objetivo de avaliar os parâmetros e limites que permitem a detecção de medicações endócrinas em testes de toxicidade específicos também podem afetar negativamente o desenvolvimento, diferenciação e reprodução de invertebrados aquáticos. Hydra vulgaris foi relatada como sendo uma das espécies mais sensíveis à toxicidade aguda e crônica do composto endócrino 4-nonilfenol em comparação com vários invertebrados de água doce (PachuraBouchet et al. 2006). Exposição de Hydra vulgaris a Bisfenol A (BPA) e 17a-etinilestradiol (EE2) levou a mudanças na estrutura dos pólipos em concentrações acima 58 e $42 \mathrm{mg} / \mathrm{L}$ de BPA e EE2, respectivamente. A regeneração foi inibida em 460 e $150 \mathrm{mg} / \mathrm{L}$ de BPA e EE2, respectivamente. Verificou-se que o efeito da exposição à EE2 prejudicava a reprodução sexual em Hydra vulgaris, em concentrações irrealistas $(500 \mathrm{mg} / \mathrm{L})$, que provavelmente, essa resposta resultou de toxicidade sistêmica em vez de interrupção dos processos hormonais/ sinalizadores (Pascoe et al. 2002).

\section{Sensibilidade a lantanídeos}

Os lantanídeos também foram testados em experimentos de ecotoxicidade com Hydra attenuata, demonstrando um razoável grau de sensibilidade. Esse grupo inclui 15 elementos químicos que ocorrem normalmente em baixas concentrações no solo e na água. Entretanto, o seu acúmulo no meio ambiente pela ação do homem pode também ter implicações prejudiciais a diversos microrganismos. Concentrações ambientais de lantanídeos podem se tornar maiores no futuro. Estudos ecotoxicológicos e de avaliação de risco, juntamente com o monitoramento, são necessários para gerenciar adequadamente estes contaminantes emergentes. (González et al. 2015).

\section{Sensibilidade às substâncias orgânicas}

As hidras têm uma menor sensibilidade aos compostos orgânicos do que aos metais e, portanto, uma limitada aplicação para testes de toxicidade de substâncias tóxicas orgânicas. Quando uma série de substâncias tóxicas orgânicos selecionados foram testados, os limites de toxicidade aguda e sub crônica para hidra foram maiores do que os valores da literatura para a maioria das espécies estudadas (Pollino \& Holdway 1999). No entanto, as exposições de Hydra attenuata a vários organofosfatos demonstraram que a hidra pode ser sensível a alguns orgânicos como pesticidas e inseticidas (Kalafatic et al. 2002).

\section{Sensibilidade a água de efluentes}

A utilização de hidras para testar o impacto ambiental tanto de efluentes residenciais e industriais também já foi avaliado mostrando bons resultados. Efluentes são uma fonte importante e altamente complexa de poluição para o meio ambiente. Devido a esta complexidade, a avaliação biológica com o uso de bio-ensaios é muito mais importante do que tão somente a realizada com produtos químicos (Quinn et al. 2012). Como parte de uma bateria de ensaios, diversas espécies de hidra foram testadas visando investigar a toxicidade a bioprodutos da indústria açucareira (Ferreira et al. 2011), aditivos para alimentação animal (Marroquin-Cardona et al. 
2009) e pesticidas (Castillo et al. 2006). Os resultados demonstraram grande sensibilidade aos produtos testados, com alterações morfológicas e no crescimento, além de letalidade dependendo do produto testado. A utilização desses organismos representou uma ferramenta poderosa de monitoramento ambiental.

\section{Considerações Finais}

Os estudos publicados que serviram de fonte para o presente trabalho de revisão destacam diversos motivos que consagraram a hidra como um modelo extremamente eficaz em estudos de toxicidade em ecossistemas aquáticos. Seu cultivo em laboratório é relativamente fácil, rápida reprodução assexuada gerando clones idênticos, estrutura diploblástica que permite a exposição de células diretamente no meio ambiente, e ainda de fácil quantificação e observação morfológica das mudanças do corpo.

As hidras fazem parte de um grupo de espécies mais sensíveis às substâncias tóxicas quando comparado a outros invertebrados, especialmente para efeitos sub letais. Além disso, o uso de animais simbióticos versus não-simbióticas pode ser muito útil para investigar os mecanismos de uma determinada toxicidade. $\mathrm{O}$ conjunto destas características permite o seu uso em um número grande de bioensaios objetivado estudar a toxicidade de um grande número de poluentes, tanto em estudos agudos como crônicos. Além disso, a hidra mostrou-se extremamente eficaz em testes de tolerância, especialmente com metais, nano particulados e compostos farmacêuticos. Alterações rápidas na morfologia do tentáculo e do processo reprodutivo e regenerativo são as principais características evidenciadas.

Entretanto, a utilização de hidras em testes com substâncias orgânicas é limitada. Seus pontos de tolerância são maiores quando comparado a maioria das espécies também utilizadas para este propósito.

\section{Referências Bibliográficas}

Ahamed M., Ali D., Alhadlaq H.A. \& Akhtar M.J. 2013. Nickel oxide nanoparticles exert cytotoxicity via oxidative stress and induce apoptotic response in human liver cells (HepG2). Chemosphere 93, 2514-22.

Browne C.L. \& Davis L.E. 1977. Cellular mechanisms of stimulation of bud production in Hydra by low levels of inorganic lead compounds. Cell and tissue research $177,555-$ 70.

Castillo L.E., Martínez E., Ruepert C., Savage C., Gilek M., Pinnock M. \& Solis E. 2006. Water quality and macroinvertebrate community response following pesticide applications in a banana plantation, Limon, Costa Rica. Science of the Total Environment 367, 418-32.

Clarke S.M., Barrick C.W. \& Samoiloff M.R. 1990. A bioassessment battery for use in an industrial setting: a new management approach. Environmental toxicology 5, 153-66.

Dias A.M.P., Brentano D.M., Carvalho-Pinto C.R.S. \& Matias W.G. 2006. Avaliação da toxicidade aguda de fluidos de corte utilizados em processos de usinagem usando como organismos-teste Poecilia reticulata e Daphnia magna. Biotemas 19, 7-13.

Fent K., Weston A.A. \& Caminada D. 2006. Ecotoxicology of human pharmaceuticals. Aquatic Toxicology 76, 122-59.

Ferreira L.F.R., Aguiar M.M., Messias T.G., Pompeu G.B., Lopez A.M.Q., Silva D.P. \& Monteiro R.T. 2011. Evaluation of sugar-cane vinasse treated with Pleurotus sajor-caju utilizing aquatic organisms as toxicological indicators. Ecotoxicology and environmental safety 74, 132-7.

Fossi M.C., Savelli C. \& Casini S. 1998. Mixed function oxidase induction in Carcinus aestuarii.: Field and experimental studies for the evaluation of toxicological risk due to Mediterranean contaminants. Comparative Biochemistry and Physiology Part $C$ : Pharmacology, Toxicology and Endocrinology 121, 321-31.

Galliot B. 2012. Hydra, a fruitful model system for 270 years. International Journal of Developmental Biology 56, 411-23.

Gonçalves S.P.C., Strauss M., Delite F.S., Clemente Z., Castro V.L. \& Martinez D.S.T. 2016. Activated carbon from pyrolysed sugarcane bagasse: Silver nanoparticle modification and ecotoxicity assessment. Science of the Total Environment 565, 833-40.

González V., Vignati D.A.L., Pons M.-N., Montarges-Pelletier E., Bojic C. \& Giamberini L. 2015. Lanthanide ecotoxicity: First attempt to measure environmental risk for aquatic organisms. Environmental pollution 199, 13947. 
Herricks E.E. \& Cairns Junior J. 1982. Biological monitoring: part III-receiving system methodology based on community structure. Water Research 16, 141-53.

Holdway D.A., Lok K. \& Semaan M. 2001. The acute and chronic toxicity of cadmium and zinc to two hydra species. Environmental toxicology 16, 557-65.

Kalafatic M., Znidaric D., Lui A. \& Wrischer M. 2002. Effect of insecticides Dimiline (WP 25, Torak EC 24 and Gamacide 20) on hydra (Hydra vulgaris Pallas). International Journal of Developmental Biology 35, 335-40.

Karntanut W. \& Pascoe D. 2002. The toxicity of copper, cadmium and zinc to four different Hydra (Cnidaria: Hydrozoa). Chemosphere 47, 1059-64.

Karntanut W. \& Pascoe D. 2005. Effects of removing symbiotic green algae on the response of Hydra viridissima (Pallas 1776) to metals. Ecotoxicology and environmental safety $60,301-5$.

Kim J., H,, Gibb H., J, \& Howe P., D, 2006. WHO Concise International Chemical Assessment. Cobalt and Inorganic Cobalt Compounds 69, 1-93.

Knie J.L.W. \& Lopes E.W.B. 2004. Testes ecotoxicológicos: métodos, técnicas e aplicações. FATMA/GTZ, Florianópolis, Santa Catarina, Brasil.

Laitano K.d.S. 2003. Testes de toxicidade com Daphnia magna: uma ferramenta para avaliação de um reator experimental UASB. Journal of the Brazilian Society of Ecotoxicology 1, 43-7.

Magalhaes D.P. \& Ferrão Filho A. 2008. A ecotoxicologia como ferramenta no biomonitoramento de ecossistemas aquáticos. Oecologia Brasiliensis 12, 355-81.

Marchesano V., Ambrosone A., Bartelmess J., Strisciante F., Tino A., Echegoyen L., Tortiglione C. \& Giordani S. 2015. Impact of carbon nano-onions on Hydra vulgaris as a model organism for nanoecotoxicology. Nanomaterials 5, 1331-50.

Marroquin-Cardona A., Deng Y., Taylor J.F., Hallmark C.T., Johnson N.M. \& Phillips T.D. 2009. In vitro and in vivo characterization of mycotoxin-binding additives used for animal feeds in Mexico. Food additives and Contaminants 26, 733-43.
Massaro F.C. \& Rocha O. 2008. Development and population growth of Hydra viridissima Pallas, 1766 (Cnidaria, Hydrozoa) in the laboratory. Brazilian Journal of Biology 68, 379-83.

Murugadas A., Zeeshan M., Thamaraiselvi K., Ghaskadbi S. \& Akbarsha M.A. 2016. Hydra as a model organism to decipher the toxic effects of copper oxide nanorod: Ecotoxicogenomics approach. Scientific Reports 6, 29663.

Pachura-Bouchet S., Blaise C. \& Vasseur P. 2006. Toxicity of nonylphenol on the cnidarian Hydra attenuata and environmental risk assessment. Environmental toxicology 21, 38894.

Pascoe D., Carroll K., Karntanut W. \& Watts M.M. 2002. Toxicity of $17 \alpha$-ethinylestradiol and bisphenol A to the freshwater cnidarian Hydra vulgaris. Archives of environmental contamination and toxicology 43, 56-63.

Pascoe D., Karntanut W. \& Müller C.T. 2003. Do pharmaceuticals affect freshwater invertebrates? A study with the cnidarian Hydra vulgaris. Chemosphere 51, 521-8.

Pollino C.A. \& Holdway D.A. 1999. Potential of two hydra species as standard toxicity test animals. Ecotoxicology and environmental safety 43, 309-16.

Quinn B., Gagné F. \& Blaise C. 2009. Evaluation of the acute, chronic and teratogenic effects of a mixture of eleven pharmaceuticals on the cnidarian, Hydra attenuata. Science of the Total Environment 407, 1072-9.

Quinn B., Gagné F. \& Blaise C. 2012. Hydra, a model system for environmental studies. International Journal of Developmental Biology 56, 613-25.

Raghunathan V.K., Devey M., Hawkins S., Hails L., Davis S.A., Mann S., Chang I.T., Ingham E., Malhas A. \& Vaux D.J. 2013. Influence of particle size and reactive oxygen species on cobalt chrome nanoparticle-mediated genotoxicity. Biomaterials 34, 3559-70.

Rand G.M., Wells P.G. \& Mccarty L.S. 1995. Introduction toaquatic toxicology. In: Fundamentals of aquatic toxicology: effects, environmental fate and risk assessment ed. (by Rand GM), North Palm Beach, Florida, USA.

Ribeiro M.L., Lourencetti C., Pereira S.Y. \& Marchi M.R.R. 2007. Contaminação de águas subterrâneas por pesticidas: avaliação preliminar. Química Nova 30, 688-94. 
Riethmuller N., Markich S.J., Van Dam R.A. \& Parry D. 2001. Effects of water hardness and alkalinity on the toxicity of uranium to a tropical freshwater hydra (Hydra viridissima). Biomarkers 6, 45-51.

Santiago-Fandino V.J.R. 1983. The effects of nickel and cadmium on the growth rate of Hydra littoralis and an assessment of the rate of uptake of $63 \mathrm{Ni}$ and $14 \mathrm{C}$ by the same organism. Water Research 17, 917-23.

Santos J.R. \& Silva J.M. 2007. Toxicologia de agrotóxicos em ambientes aquáticos. Oecologia Brasiliensis 11, 565-73.

Schaible R., Sussman M. \& Kramer B.H. 2014. Aging and potential for self-renewal: hydra living in the age of aging-a mini-review. Gerontology 60, 548-56.

Ten Brink B.J.E. \& Woudstra J.H. 1991. Towards an effective and rational water management: The aquatic outlook project - Integrating water management, monitoring and research. European Water Pollution Control 1, 20-7.

Trottier S., Blaise C., Kusui T. \& Johnson E.M. 1997. Acute toxicity assessment of aqueous samples using a microplate-based Hydra attenuata assay. Environmental Toxicology and
Water Quality: An International Journal 12, 265-71.

Trouiller B., Reliene R., Westbrook A., Solaimani P. \& Schiestl R.H. 2009. Titanium dioxide nanoparticles induce DNA damage and genetic instability in vivo in mice. Cancer research 69 , 8784-9.

Zeeshan M., Murugadas A., Ghaskadbi S., Ramaswamy B.R. \& Akbarsha M.A. 2017. Ecotoxicological assessment of cobalt using Hydra model: ROS, oxidative stress, DNA damage, cell cycle arrest, and apoptosis as mechanisms of toxicity. Environmental pollution 224, 54-69.

Article History:

Received 12 April 2018

Accepted 2 May 2018

Available online 28 June 2018

License information: This is an open-access article distributed under the terms of the Creative Commons Attribution License 4.0, which permits unrestricted use, distribution, and reproduction in any medium, provided the original work is properly cited. 